\section{Effectiveness of a Hydrogen Peroxide Mist (Trophon) System in Inactivating Healthcare Pathogens on Surface and Endocavitary Probes}

Ultrasound probes are used in sonographic scanning and are commonly used as either surface probes or endocavitary probes. Surface probes are used on intact skin, such as the abdomen; they are considered noncritical and require at least low-level disinfection between patients. Endocavitary probes (eg, transvaginal, transrectal, or transesophageal probes) are considered semicritical because they have direct contact with mucous membranes (eg, vagina, rectum, or pharynx) or nonintact skin. While one could argue that the use of the probe cover changes the category for the endocavitary probe, the Centers for Disease Control and Prevention (CDC) guideline for disinfection and sterilization recommends that a new condom/probe cover should be used for each patient, and because condoms/probe covers and low-level disinfection may fail, ${ }^{1,2}$ high-level disinfection of the probe should be performed. ${ }^{3}$ The relevance of this recommendation is reinforced by the finding that sterile ultrasound probe covers and condoms can have a perforation rate from $0 \%$ to $81 \%$ before and after use. ${ }^{1}$ These studies underscore the need for high-level disinfection of endocavitary probes between examinations.

Ultrasound probes may also be used during surgical procedures and may have contact with sterile body sites. These probes may be covered with a sterile sheath to reduce the level of contamination on the probe and reduce the risk of infection. However, because the sheath does not provide complete protection of the probe, the probes should be sterilized between each patient use, as are other critical items. If this is not possible, then highlevel disinfection of the probe should be performed and the probe should be covered with a sterile probe cover. ${ }^{1,3}$

Although the most common method of performing high-level disinfection of contaminated endocavitary probes is by immersion in a high-level disinfectant cleared by the Food and Drug Administration (eg, glutaraldehyde), an alternative procedure for disinfecting the endocavitary and surface probes uses a proprietary hydrogen peroxide mist system, which utilizes 35\% hydrogen peroxide at $56^{\circ} \mathrm{C}$ with the probe reaching no more than $40^{\circ} \mathrm{C}$ (ie, Trophon EPR, Nanosonics, Alexandria, Australia). The effectiveness of this technology, which has been cleared by the Food and Drug Administration for high-level disinfection, is evaluated in this research brief.

This study was conducted at the University of North Carolina (UNC) Hospitals, an 853-bed academic medical center. Trophon EPR and 5 ultrasound probes were loaned to UNC Hospitals for use in the study. In a biological safety cabinet, at least 3 replicates of each probe type (ie, endocavitary or surface probe) were inoculated in 3 specified locations with one of the test organisms: vancomycin-resistant Enterococcus [VRE] ATCC \#51299, a clinical strain of carbapenem-resistant Enterobacteriaceae [CRE] Klebsiella pneumoniae, Clostridium difficile spores, or Mycobacterium terrae. These procedures were fully repeated in a separate experiment to ensure accuracy using the aforementioned defined set of test conditions. The inoculae for VRE, CR-K. pneumoniae, M. terrae, and C. difficile spores in the presence of $5 \%$ fetal calf serum (FCS) produced inoculation levels of 5.59- $\log _{10}, 5.91-\log _{10}$, $5.88-\log _{10}$, and $6.23-\log _{10}$, respectively, and in the absence of $5 \%$ FCS they produced inoculation levels of $5.84-\log _{10}$, $6.16-\log _{10}, 4.89-\log _{10}$, and $6.29-\log _{10}$, respectively. In total, 24 surface probes and 31 endocavitary probes were tested. The presence and resistance of $C$. difficile spores (and not vegetative bacteria) were verified by exposing the stock preparation to dilute hydrochloric acid as specified in the AOAC International sporicidal activity test. ${ }^{4}$

The $15 \mu \mathrm{L}$ inoculum consisted of trypticase soy broth (TSB; Becton, Dickinson and Company, Sparks, MD) with or without 5\% FCS (Remel, Lenexa, KS) as an organic challenge. Each inoculum was spread over an area equal to the size of a dime to prevent pooling of the inoculum. The probes were then allowed to air dry for 30 minutes. After drying, each test probe was processed in the Trophon EPR according to the manufacturer's instructions. A chemical indicator was used in each cycle to ensure that the critical parameters of the cycle had been met. After processing, the probe was evaluated for surviving microbes by submerging the inoculated portion of the probe in TSB ( $\sim 400 \mathrm{~mL}$ surface, $\sim 1500 \mathrm{~mL}$ endocavitary) and shaking it at $80-100 \mathrm{rpm}$ (Fisher Scientific Clinical Rotator, Pittsburgh, PA) for a minimum of 1 hour. After shaking, the TSB for each probe was filtered aseptically $(0.2 \mu$ pore size, Thermo Scientific, Waltham, MA), and the filter was removed and placed on media appropriate for each test organism. VRE, CR Klebsiella pneumoniae, and C. difficile were all plated to sheep blood agar, and Mycobacterium terrae was plated to Middlebrook 7H11 Agar. All plates were then incubated at $35-37^{\circ} \mathrm{C}$ in a manner appropriate for each test organism: aerobically for 48 hours for VRE and Klebsiella pneumoniae; anaerobically for 48-96 hours for $C$. difficile; and aerobically for 28 days for Mycobacterium terrae.

The results demonstrated complete inactivation $\left(>6-\log _{10}\right.$ reduction) of VRE and a CR-K. pneumoniae strain both in the presence and absence of 5\% FCS (Table 1). The Trophon EPR system showed good, but not complete, inactivation of $M$. terrae (a $5.2-\log _{10}$ reduction for M. terrae with FCS and a $4.6-\log _{10}$ reduction for M. terrae without FCS) and C. difficile (a $5.1-\log _{10}$ reduction for $C$. difficile spores with FCS and a $6.2-\log _{10}$ reduction for $C$. difficile spores without FCS spores) (Table 1). To simulate a worst-case condition, cleaning was not done prior to disinfection in these experiments, 
table 1. Proportion of Surface and Endocavitary Probes Positive After Trophon System Processing According to the Presence or Absence of an Organic Challenge*

\begin{tabular}{lcccc}
\hline $\begin{array}{l}\text { 5\% Fetal Calf } \\
\text { Serum-FCS }\end{array}$ & $\begin{array}{c}\text { Probes with vancomycin- } \\
\text { Resistant Enterococcus } \\
\text { (VRE), No./Total }\end{array}$ & $\begin{array}{c}\text { Probes with CR Klebsiella } \\
\text { pneumoniae, No./Total }\end{array}$ & $\begin{array}{c}\text { Probes with Mycobacterium } \\
\text { terrae, No./Total (mean } \log _{10} \\
\text { reduction and 95\% CI) }\end{array}$ & $\begin{array}{c}\text { Probes with Clostridium difficile } \\
\text { spores, No./Total (mean log } \\
\text { reductions and 95\% CI) }\end{array}$ \\
\hline Present & $0 / 7$ & $0 / 6$ & $4 / 9(5.19[4.61-5.76])$ & $3 / 6(5.12[4.42-5.83])$ \\
Absent & $0 / 6$ & $0 / 6$ & $1 / 6(4.62[4.07-5.17])$ & $1 / 9(6.23[6.02-6.43])$ \\
\hline
\end{tabular}

${ }^{\star}$ The inoculum control was handled like the test but not processed in the Trophon. Numbers represent a proportion of the number of probes positive with the test organisms per number of probes tested. No statistical difference in the proportion of positive probes by test organism with and without fetal calf serum (Fisher's exact test; $P>.05$ ).

but proper cleaning of probes is necessary to ensure the success of high-level disinfection. Other pathogens that could contaminate vaginal and rectal probes include human papilloma virus. ${ }^{5}$ Published data have demonstrated the activity of Trophon to inactivate $\mathrm{HPV}^{6}$ and other pathogens (eg, bacteria, mycobacteria, and viruses), including a $>6-\log _{10}$ reduction of $M$. terrae and $C$. difficile spores in carrier tests and a $>6-\log _{10}$ reduction in $M$. terrae on inoculated ultrasound probes. ${ }^{7}$ These results differ slightly from those presented here, presumably due to the differences in testing methodology. In our study, only the probe devices were inoculated (ie, carriers of different materials were not tested), and for recovery of bacteria on the probe, the probes were immersed in media (ie, not swabbed, ${ }^{7}$ which would likely result in a lower recovery rate).

The Trophon system processes the portion of the probe that has contact not only with mucous membrane but also with the handle of an endocavitary probes, which also may be contaminated. ${ }^{8}$ It is an alternative to high-level chemical disinfection for ultrasound probes.

\section{ACKNOWLEDGMENTS}

Financial support: Nanosonics provided funding to UNC Hospitals for this study.

Potential conflicts of interest: Dr. Rutala is a consultant for Clorox and was a consultant for ASP in 2014. He has received honoraria from 3M. The other authors report no conflicts of interest relevant to this article.

\section{William A. Rutala, PhD, MPH; ${ }^{1,2}$ Maria F. Gergen, MT (ASCP); ${ }^{1}$ Emily E. Sickbert-Bennett, $\mathrm{PhD}^{1,2}$}

Affiliations: 1. Hospital Epidemiology, University of North Carolina Health Care, Chapel Hill, North Carolina; 2. Division of Infectious Diseases, UNC School of Medicine, Chapel Hill, North Carolina.

Address correspondence to William A. Rutala, PhD, MPH, Director, Hospital Epidemiology, Occupational Health, and Safety Program, Room 1001 West Wing, UNC Health Care Chapel Hill, NC 27514 (brutala@unch.unc.edu).

Received August 9, 2015; accepted January 11, 2016; electronically published February 4, 2016

Infect Control Hosp Epidemiol 2016;37:613-614

(C) 2016 by The Society for Healthcare Epidemiology of America. This is an Open Access article, distributed under the terms of the Creative Commons Attribution licence (http://creativecommons.org/licenses/by/4.0/), which permits unrestricted re-se, distribution, and reproduction in any medium, provided the original work is properly cited. All rights reserved. 0899-823X/2016/3705-0020. DOI: $10.1017 /$ ice.2016.11

\section{REFERENCES}

1. Rutala WA, Weber DJ. 2013. New developments in reprocessing semicritical items. Am J Infect Control 41:S60-S66.

2. M'Zali F, Bounizra C, Leroy S, Mekki Y, Quentin-Noury C. Persistence of microbial contamination on transvaginal probes despite low-level disinfection. PLoS One 2014;9:e93368.

3. Rutala WA, Weber DJ. Healthcare Infection Control Practices Advisory Committee. Guideline for disinfection and sterilization in healthcare facilities, 2008. Centers for Disease Control and Prevention website. http://www.cdc.gov/hicpac/pdf/guidelines/ Disinfection_Nov_2008.pdf. Published 2008. Accessed 10 September 2015.

4. Beloian A. Disinfectants. In: Cunniff P, ed. Official Methods of Analysis of the AOAC International, Vol 1. Gaithersburg, Maryland: AOAC International, 1999:6-1; 6-18.

5. Casalegno J-S, Carval KLB, Eibach D, et al. High-risk HPV contamination of endocavitary vaginal ultrasound probes: an underestimated route of nosocomial infection? PLoS One 2012;7: e48137.

6. Ryndock E, Robison R, Meyers C. Susceptibility of HPV 16 and 18 to high-level disinfectants indicated for semi-critical ultrasound probes. J Med Virol 2015, October Epub ahead of print.

7. Vickery K, Gorgis VZ, Burdach J, Patel D. Evaluation of an automated high-level disinfection technology for ultrasound transducers. J Infect Public Health 2014;7:153-160.

8. Ngu A, McNally G, Patel D, Leroy S, Burdach J. Reducing transmission risk through high-level disinfection of transvaginal ultrasound transducer handles. Infect Control Hosp Epidemiol 2015;36:581-584.

\section{Multidrug-Resistant Organism Colonization in a High-Risk Pediatric Patient Population}

Multidrug-resistant organisms (MDROs) present substantial clinical and financial burdens to patients and hospitals. In pediatric patients undergoing cancer or hematopoietic stem cell treatment, information is needed to characterize the 